\begin{tabular}{cc} 
Sharif University of Technology \\
Scientia Iranica \\
SCIENTIA & Transactions B: Mechanical Engineering \\
I RAN I CA & \\
\hline
\end{tabular}

\title{
RPT finite-element formulation for linear dynamic analysis of orthotropic plates
}

\author{
J. Rouzegar* and M. Sayedain \\ Department of Mechanical and Aerospace Engineering, Shiraz University of Technology, Shiraz, P.O. Box 71555-313, Iran.
}

Received 21 November 2016; received in revised form 10 January 2017; accepted 6 March 2017

\section{KEYWORDS}

Orthotropic;

Finite-element

method;

Dynamic analysis;

Two-variable refined

plate theory;

Rectangular plate

element.

\begin{abstract}
This paper presents a finite-element formulation for dynamic analysis of orthotropic plates using two-variable Refined Plate Theory (RPT). Hamilton's principle is employed to obtain the governing equations, and the semi-discrete approach is utilized for solving the equations. After constructing spatial weak form equations, a 4-node rectangular plate element with six Degrees Of Freedom (DOFs) per node is introduced for discretization of the domain. An unconditionally stable implicit Newmark scheme is used for temporal discretization. A MATLAB code with the capability of modelling both static and dynamic plate problems with various boundary conditions is generated. Several numerical problems are solved, and the obtained displacements and stresses are compared with the existing results in the literature. The results demonstrate the accuracy, simplicity, and efficiency of the present method in dynamic analysis of plate problems.
\end{abstract}

(C) 2018 Sharif University of Technology. All rights reserved.

\section{Introduction}

Plates are structural elements commonly used in many industrial applications, e.g. aerospace, marine, nuclear industries, etc. They are classified in the literature as thin, moderately thick, or thick plate. The classification of a rectangular plate into one of the three aforementioned categories is based on relation $h / a: h$ being the plate thickness and $a$ being the shortest side length.

In the framework of Classical Plate Theory (CPT) [1], which is the simplest plate theory, the results are unrealistic in the case of thick plates because it does not take into account shear deformation effects. First-order Shear Deformation plate Theory (FSDT) presented by Mindlin [2] and Reissner [3] considers the shear effects in its formulation, but it needs a

\footnotetext{
*. Corresponding author. Tel./Fax: +98 7137264102
} E-mail address: rouzegar@sutech.ac.ir (J.Rouzegar) shear correction factor to refine the overestimated shear strain energy of the plate. Another drawback to this theory is the constant transverse shear stress across the plate thickness which is inconsistent with stressfree conditions on the plate surfaces. Higher-order Shear Deformation Theories (HSDTs) were developed using more unknown variables in their formulations to overcome shortcomings of CPT and FSDT [4-10].

Recently, several simple and efficient HSDTs have been proposed and employed in study of various structures such as functionally graded, laminated, and sandwich plates and shells [11-13]. In 2002, Shimpi [13] presented a simple HSDT called two-variable Refined Plate Theory (RPT), which involves only two unknown functions. He separated the transverse deflection into the shear and bending parts which resulted in two uncoupled governing equations for bending analysis of isotropic plates. The two-variable refined plate theory, which can be used for thin and thick plates, predicts parabolic variation of transverse shear stresses across the plate thickness and does not need shear correction factor. In this theory, zero traction conditions are 
satisfied on the plate surfaces. This theory was rapidly developed and extended to various applications. In 2006, Shimpi and Patel [14] employed this this method for analysis of orthotropic plates. In 2009, Kim et al. [15] extended the application of this theory to analysis of laminated composite plate. They investigated the bending of antisymmetric cross-ply and angle-ply laminates using Navier solution. In 2012, Thai and Kim [16] used the two-variable refined plate theory, Levy-type solution procedure, and state space concept to obtain a closed-form solution for orthotropic rectangular plates with two opposite edges simply supported and the other two edges having arbitrary boundary conditions. Rouzegar and Abdoli presented finite-element formulations for free vibration [17] and buckling [18] analysis of isotropic and orthotropic plates using this theory. By adding two in-plane displacements to this theory, a four-variable refined plate theory was introduced. Rouzegar and Abad employed this theory for bending [19] and free vibration [20] analysis of cross-ply laminated plates integrated with piezoelectric layers. Rouzegar and Gholami [21] studied the non-linear bending analysis of rectangular plates by four-variable refined plate theory and Dynamic relaxation method.

The practical and typically complicated problems could be solved in an approximate manner employing various numerical approaches, such as finite-layer method, collocation method, finite-element method, finite strip method, and meshless methods. Among different numerical approaches, Finite-Element Method (FEM) is an efficient and attractive technique established in the 1940's. Recently, some investigations have been performed on isogeometric finite-element approach utilizing shear deformation plate theories. Nguyen-Xuan et al. [22] employed isogeometric finiteelement method with a refined plate theory for static, free vibration and buckling analysis of FG plates. Thai et al. [23] presented a generalized shear deformation theory for static, dynamic and buckling analysis of isotropic and sandwich FG plates. An inverse trigonometric shear deformation theory along with an isogeometric approach was employed in this study. Nguyen and Nguyen-Xuan [24] presented an isogeometric finite-element approach to three-dimensional static and dynamic analyses of FG plates. Nguyen et al. [25] introduced a novel unified framework on HSDTs for the modeling and analysis of laminated composite plates. They found that the proposed formulation with a polynomial form can theoretically cover all existing HSDTs models and is thus sufficient to describe the nonlinear and parabolic variations of transverse shear stress. Nguyen et al. [26] presented an isogeometric finite-element formulation based on Bézier extraction of the non-uniform rational B-splines in combination with a generalized unconstrained higher- order shear deformation theory for laminated composite plates.

Previous researches on two-variable refined plate theory are mostly focused on analytical solutions to some plate problems with specific geometry, loading and boundary conditions. A new finite-element formulation of shear deformation plate was introduced by $\mathrm{Pa}$ tel and Shimpi [27]. Katori and Okada [28] developed a finite-element formulation based on the refined plate theory using triangular and quadrilateral elements for discretization of the domain. Recently, Rouzegar and Abdoli [29] presented a finite-element formulation for static analysis of isotropic and orthotropic plates using a two-variable refined plate theory.

Although dynamic analysis of plate structures is the subject of a large number of research works, there are still some hypotheses either not introduced or not sufficiently tested. In this study, a new finite-element formulation based on the two-variable refined plate theory is developed for dynamic analysis of isotropic and orthotropic plates. The semi-discrete method is used to simulate the dynamic behavior of problems, and an implicit Newmark scheme is employed for temporal discretization. The performance of the code in simulation of isotropic and orthotropic plates under static and dynamic loadings is proved by solving several benchmark problems.

\section{Theory of problem}

\subsection{Two-variable refined plate theory}

Consider a homogeneous, orthotropic, thick elastic plate of thickness, $h$, which is subjected to a transverse dynamic load, $q(x, y, t)$, per unit area. According to Figure 1 , axes $x, y$, and $z$ are the Cartesian coordinate systems located at the corner of the plate.

Based on two-variable refined plate theory, the displacement field is assumed as follows [13]:

$$
\begin{aligned}
& u(x, y, z, t)=-z \frac{\partial w_{b}}{\partial x}-f(z) \frac{\partial w_{s}}{\partial x} \\
& v(x, y, z, t)=-z \frac{\partial w_{b}}{\partial y}-f(z) \frac{\partial w_{s}}{\partial y} \\
& w(x, y, t)=w_{b}(x, y, t)+w_{s}(x, y, t)
\end{aligned}
$$

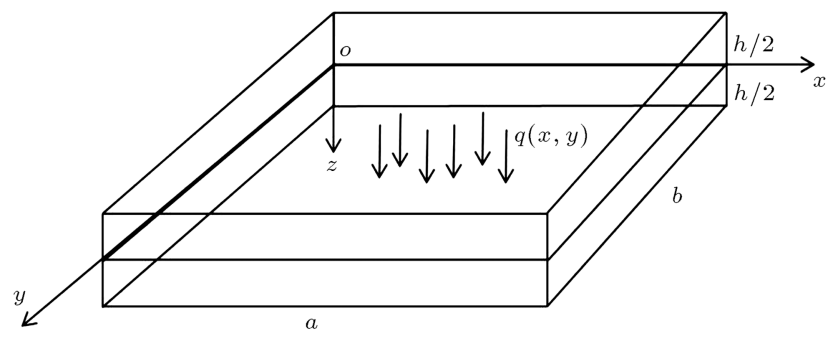

Figure 1. Geometry and coordinate system of the problem. 
where $u$ and $v$ are the displacements in $x$ and $y$ directions, $w_{b}$ and $w_{s}$ are bending and shear components of transverse displacement $w$, and:

$$
f(z)=-\frac{z}{4}+\frac{5 z}{3}\left(\frac{z}{h}\right)^{2} .
$$

Regarding the assumption of small displacements, the infinitesimal strain components are obtained as follows:

$$
\begin{aligned}
& \varepsilon_{x}=z \kappa_{x}^{b}+f(z) \kappa_{x}^{s}, \\
& \varepsilon_{y}=z \kappa_{y}^{b}+f(z) \kappa_{y}^{s}, \\
& \gamma_{x y}=z \kappa_{x y}^{b}+f(z) \kappa_{x y}^{s}, \\
& \gamma_{y z}=g(z) \gamma_{y z}^{s}, \\
& \gamma_{x z}=g(z) \gamma_{x z}^{s}, \\
& \varepsilon_{z}=0,
\end{aligned}
$$

where:

$$
\begin{aligned}
& \kappa_{x}^{b}=-\frac{\partial^{2} w_{b}}{\partial x^{2}}, \quad \kappa_{x}^{s}=-\frac{\partial^{2} w_{s}}{\partial x^{2}}, \\
& \kappa_{y}^{b}=-\frac{\partial^{2} w_{b}}{\partial y^{2}}, \quad \kappa_{y}^{s}=-\frac{\partial^{2} w_{s}}{\partial y^{2}}, \\
& \kappa_{x y}^{b}=-2 \frac{\partial^{2} w_{b}}{\partial x \partial y}, \quad \kappa_{x y}^{s}=-2 \frac{\partial^{2} w_{s}}{\partial x \partial y} \\
& \gamma_{x z}^{s}=\frac{\partial w_{s}}{\partial x}, \quad \gamma_{y z}^{s}=\frac{\partial w_{s}}{\partial y}, \\
& g(z)=1-\frac{d f(z)}{d z}=\left[\frac{5}{4}-5\left(\frac{z}{h}\right)^{2}\right] .
\end{aligned}
$$

Neglecting normal stress, $\sigma_{z}$, the other stress components for orthotropic plate are obtained by the following constitutive relations:

$$
\begin{gathered}
\left\{\begin{array}{l}
\sigma_{x} \\
\sigma_{y} \\
\tau_{x y} \\
\tau_{y z} \\
\tau_{z x}
\end{array}\right\}= \\
{\left[\begin{array}{ccccc}
Q_{11} & Q_{12} & 0 & 0 & 0 \\
Q_{12} & Q_{22} & 0 & 0 & 0 \\
0 & 0 & Q_{66} & 0 & 0 \\
0 & 0 & 0 & Q_{44} & 0 \\
0 & 0 & 0 & 0 & Q_{55}
\end{array}\right]\left\{\begin{array}{l}
\varepsilon_{x} \\
\varepsilon_{y} \\
\gamma_{x y} \\
\gamma_{y z} \\
\gamma_{z x}
\end{array}\right\}}
\end{gathered}
$$

where:

$$
Q_{11}=\frac{E_{1}}{1-\mu_{12} \mu_{21}},
$$

$$
\begin{aligned}
& Q_{12}=\frac{\mu_{12} E_{2}}{1-\mu_{12} \mu_{21}}=\frac{\mu_{21} E_{2}}{1-\mu_{12} \mu_{21}}, \\
& Q_{22}=\frac{E_{2}}{1-\mu_{12} \mu_{21}}, \quad Q_{44}=G_{23}, \\
& Q_{55}=G_{31}, \quad Q_{66}=G_{12},
\end{aligned}
$$

where $E_{1}, E_{2}, G_{12}, G_{23}$, and $G_{31}$ are elastic moduli, and $\mu_{12}$ and $\mu_{21}$ are Poisson's ratios.

\subsection{Governing equations for dynamic problems}

The governing equations and boundary conditions are obtained using Hamilton's principle:

$$
0=\int_{t_{1}}^{t_{2}} \delta(T-\Pi) d t .
$$

Total potential energy of the plate, $\Pi$, is the sum of strain energy and work done by external loads:

$$
\begin{aligned}
\Pi= & \int_{z=-h / 2}^{z=h / 2} \int_{y=0}^{y=b} \int_{x=0}^{x=a} \frac{1}{2}\left[\sigma_{x} \varepsilon_{x}+\sigma_{y} \varepsilon_{y}+\tau_{x y} \gamma_{x y}\right. \\
& \left.+\tau_{y z} \gamma_{y z}+\tau_{z x} \gamma_{z x}\right] d x d y d z+\int_{0}^{a} \int_{0}^{b} q w d x d y,
\end{aligned}
$$

where $q$ is the normal external pressure applied onto the top surface of plate. Total kinetic energy of the plate $(T)$ can be written as follows:

$$
\begin{aligned}
T= & \int_{z=-h / 2}^{z=h / 2} \int_{y=0}^{y=b} \int_{x=0}^{x=a} \frac{1}{2} \rho\left[\left(\frac{\partial u}{\partial t}\right)^{2}+\left(\frac{\partial v}{\partial t}\right)^{2}\right. \\
& \left.+\left(\frac{\partial w}{\partial t}\right)^{2}\right] d x d y d z .
\end{aligned}
$$

By substituting stress, strain, and displacement fields into Eqs. (6)-(8) and taking into account the independent variations of $w_{b}$ and $w_{s}$, the governing differential equations are obtained as follows:

$$
\begin{gathered}
D_{11} \frac{\partial^{4} w_{b}}{\partial x^{4}}+2\left(D_{12}+2 D_{66}\right) \frac{\partial^{4} w_{b}}{\partial x^{2} \partial y^{2}}+D_{22} \frac{\partial^{4} w_{b}}{\partial y^{4}} \\
-\frac{\rho h^{3}}{12} \frac{\partial^{2}}{\partial t^{2}}\left(\nabla^{2} w_{b}\right)+\rho h\left(\frac{\partial^{2} w_{b}}{\partial t^{2}}+\frac{\partial^{2} w_{s}}{\partial t^{2}}\right)=q \\
\frac{1}{84}\left[D_{11} \frac{\partial^{4} w_{s}}{\partial x^{4}}+2\left(D_{12}+2 D_{66}\right) \frac{\partial^{4} w_{s}}{\partial x^{2} \partial y^{2}}+D_{22} \frac{\partial^{4} w_{s}}{\partial y^{4}}\right] \\
-\left[A_{55} \frac{\partial^{2} w_{s}}{\partial x^{2}}+A_{44} \frac{\partial^{2} w_{s}}{\partial y^{2}}\right]-\frac{\rho h^{3}}{1008} \frac{\partial^{2}}{\partial t^{2}}\left(\nabla^{2} w_{s}\right) \\
+\rho h\left(\frac{\partial^{2} w_{b}}{\partial t^{2}}+\frac{\partial^{2} w_{s}}{\partial t^{2}}\right)=q,
\end{gathered}
$$


where $D_{11}, D_{22}, D_{12}, D_{66}, A_{44}$, and $A_{55}$ are material stiffnesses of the plate:

$$
\begin{aligned}
& D_{11}=\frac{Q_{11} h^{3}}{12}, \quad D_{22}=\frac{Q_{22} h^{3}}{12}, \\
& D_{12}=\frac{Q_{12} h^{3}}{12}, \quad D_{66}=\frac{Q_{66} h^{3}}{12}, \\
& A_{44}=\frac{5 Q_{44} h}{6}, \quad A_{55}=\frac{5 Q_{55} h}{6} .
\end{aligned}
$$

\subsection{Finite-element formulation for dynamic problems}

Appling the Hamilton's principle for a plate element with volume, $v_{e}$, and mid-plane surface, $\Omega_{e}$, the following equation will be obtained:

$$
\begin{gathered}
\frac{1}{2} \int_{v_{e}} \delta\left[\left(\frac{\partial u}{\partial t}\right)^{2}+\left(\frac{\partial v}{\partial t}\right)^{2}+\left(\frac{\partial w}{\partial t}\right)^{2}\right] d v \\
-\frac{1}{2} \int_{v_{e}} \delta\left(\sigma_{x x} \varepsilon_{x x}+\sigma_{y y} \varepsilon_{y y}+\sigma_{x y} \gamma_{x y}\right. \\
\left.+\sigma_{x z} \gamma_{x z}+\sigma_{y z} \gamma_{y z}\right) d v-\int_{\Omega_{e}} q \delta w d x d y \\
-\oint_{\Gamma_{e}}\left(-M_{n n} \frac{\partial \delta w}{\partial n}+V_{n} \delta w\right) d s=0
\end{gathered}
$$

where $V_{n}$ and $M_{n n}$ are the effective shear force and bending moment, respectively. Substituting the stress and strain and displacements fields into Eq. (12), we will have:

$$
\begin{aligned}
\delta(T & -U)=\frac{\rho h^{3}}{12} \int_{\Omega_{e}}\left(\frac{\partial^{3} w_{b}}{\partial x \partial t^{2}} \frac{\partial \delta w_{b}}{\partial x}+\frac{\partial^{3} w_{b}}{\partial y \partial t^{2}} \frac{\partial \delta w_{b}}{\partial y}\right) d x d y \\
& +\frac{\rho h^{3}}{1008} \int_{\Omega_{e}}\left(\frac{\partial^{3} w_{s}}{\partial x \partial t^{2}} \frac{\partial \delta w_{s}}{\partial x}+\frac{\partial^{3} w_{s}}{\partial y \partial t^{2}} \frac{\partial \delta w_{s}}{\partial y}\right) d x d y \\
& +\int_{\Omega_{e}}\left[\left(D_{11} \frac{\partial^{2} w_{b}}{\partial x^{2}}+D_{12} \frac{\partial^{2} w_{b}}{\partial y^{2}}\right) \frac{\partial^{2} \delta w_{b}}{\partial x^{2}}\right. \\
& \left.+\int_{12} \frac{\partial^{2} w_{b}}{\partial x^{2}}+D_{22} \frac{\partial^{2} w_{b}}{\partial y^{2}}\right) \frac{\partial^{2} \delta w_{b}}{\partial y^{2}} \\
+ & \left.4 D_{66} \frac{\partial^{2} w_{b}}{\partial x \partial y} \frac{\partial^{2} \delta w_{b}}{\partial x \partial y}\right] d x d y \\
+ & \int_{\Omega_{e}}\left\{\frac { 1 } { 8 4 } \left[\left(D_{11} \frac{\partial^{2} w_{s}}{\partial x^{2}}+D_{12} \frac{\partial^{2} w_{s}}{\partial y^{2}}\right) \frac{\partial^{2} \delta w_{s}}{\partial x^{2}}\right.\right.
\end{aligned}
$$

$$
\begin{aligned}
& +\left(D_{12} \frac{\partial^{2} w_{s}}{\partial x^{2}}+D_{22} \frac{\partial^{2} w_{s}}{\partial y^{2}}\right) \frac{\partial^{2} \delta w_{s}}{\partial y^{2}} \\
& \left.+4 D_{66} \frac{\partial^{2} w_{s}}{\partial x \partial y} \frac{\partial^{2} \delta w_{s}}{\partial x \partial y}\right]+\left(A_{44} \frac{\partial w_{s}}{\partial x} \frac{\partial \delta w_{s}}{\partial x}\right. \\
& \left.\left.+A_{55} \frac{\partial w_{s}}{\partial y} \frac{\partial \delta w_{s}}{\partial y}\right)\right\} d x d y+\rho h \int_{\Omega_{e}}\left[\frac { \partial } { \partial t } \left(\delta w_{b}\right.\right. \\
& \left.\left.+\delta w_{s}\right)\left(\frac{\partial w_{b}}{\partial t}+\frac{\partial w_{s}}{\partial t}\right)\right] d x d y \\
& -\int_{\Omega_{e}} q \delta\left(w_{b}+w_{s}\right) d x d y+\oint_{\Gamma_{e}}\left[-M_{n n} \frac{\partial \delta\left(w_{b}+w_{s}\right)}{\partial n}\right. \\
& \left.+V_{n} \delta\left(w_{b}+w_{s}\right)\right] d s .
\end{aligned}
$$

The weak form of governing equations is obtained by equating variation of sum of kinetic and total potential energies to zero:

$$
\begin{aligned}
& \int_{\Omega^{e}}\left[I_{0}\left(\delta w_{b}\right)^{T} \ddot{w}_{b}+I_{2}\left(D_{1} \delta w_{b}\right)^{T}\left(D_{1} \ddot{w}_{b}\right)+I_{0}\left(\delta w_{s}\right)^{T} \ddot{w}_{s}\right. \\
& \left.+\frac{I_{2}}{84}\left(D_{1} \delta w_{s}\right)^{T}\left(D_{1} \ddot{w}_{s}\right)\right] d x d y \\
& +\int_{\Omega^{e}}\left[I_{0}\left(\ddot{w}_{b}+\ddot{w}_{s}\right) \delta\left(w_{b}+w_{s}\right)\right] d x d y \\
& +\int_{\Omega^{e}}\left[\left(\left(D_{2} \delta w_{b}\right)^{T} D\left(D_{2} w_{b}\right)\right)\right. \\
& +\left(\frac{1}{84}\left(D_{2} \delta w_{s}\right)^{T} D\left(D_{2} w_{s}\right)\right. \\
& \left.\left.+\left(D_{1} \delta w_{s}\right)^{T} A\left(D_{1} w_{s}\right)\right)\right] d x d y \\
& -\int_{\Omega^{e}}\left[\delta\left(w_{b}+w_{s}\right)^{T} q\right] d x d y \\
& -\oint_{\Gamma_{e}}\left\{-\left[\frac{\delta\left(\partial\left(w_{b}+w_{s}\right)\right.}{\partial n}\right]^{T} M_{n n}\right. \\
& \left.+\left[\delta\left(w_{b}+w_{s}\right)\right]^{T} V_{n}\right\} d s=0,
\end{aligned}
$$

in which $I_{0}, I_{2}, A, D, D_{1}$, and $D_{2}$ are:

$$
I_{0}=\rho h, \quad I_{2}=\frac{\rho h^{3}}{12},
$$




$$
\begin{array}{ll}
A=\left[\begin{array}{cc}
A_{44} & 0 \\
0 & A_{55}
\end{array}\right], & D=\left[\begin{array}{ccc}
D_{11} & D_{12} & 0 \\
D_{12} & D_{22} & 0 \\
0 & 0 & D_{66}
\end{array}\right], \\
D_{1}=\left[\begin{array}{ll}
\frac{\partial}{\partial x} & \frac{\partial}{\partial y}
\end{array}\right]^{T}, & D_{2}=\left[\begin{array}{lll}
\frac{\partial^{2}}{\partial x^{2}} & \frac{\partial^{2}}{\partial y^{2}} & 2 \frac{\partial^{2}}{\partial x \partial y}
\end{array}\right]^{T} .
\end{array}
$$

In each element, the bending and shear components of transverse displacement are computed as follows:

$$
\begin{aligned}
& w_{b}(x, y)=\sum_{j=1}^{n} \Delta_{j}^{b} \varphi_{j}(x, y)=N^{T} \Delta_{b}, \\
& w_{s}(x, y)=\sum_{j=1}^{n} \Delta_{j}^{s} \varphi_{j}(x, y)=N^{T} \Delta_{s},
\end{aligned}
$$

where $\Delta_{b}, \Delta_{s}, \varphi_{j}$, and $N$ are bending DOFs, shear DOFs, interpolating functions, and shape functions, respectively. The two-variable RPT finite-element equations are obtained by substituting Eq. (16) into Eq. (14):

$$
\begin{aligned}
& {\left[\begin{array}{ll}
M^{11} & M^{12} \\
M^{12} & M^{22}
\end{array}\right]\left\{\begin{array}{l}
. \\
\Delta_{b} \\
\ddot{\Delta_{s}}
\end{array}\right\}+\left[\begin{array}{cc}
K^{11} & 0 \\
0 & K^{22}
\end{array}\right]\left\{\begin{array}{l}
\Delta_{b} \\
\Delta_{s}
\end{array}\right\}} \\
& \quad=\left\{\begin{array}{l}
F \\
F
\end{array}\right\},
\end{aligned}
$$

where:

$$
\begin{aligned}
& K^{11}=\int_{\Omega_{e}}\left(B_{2}{ }^{T} D B_{2}\right) d x d y, \\
& K^{22}=\int_{\Omega_{e}}\left(\frac{1}{84} B_{2}{ }^{T} D B_{2}+B_{1}{ }^{T} A B_{1}\right) d x d y, \\
& M^{11}=\int_{\Omega_{e}}\left(I_{0} N N^{T}+I_{2} B_{1}{ }^{T} B_{1}\right) d x d y, \\
& M^{22}=\int_{\Omega_{e}}\left(I_{0} N N^{T}+\frac{I_{2}}{84} B_{1}{ }^{T} B_{1}\right) d x d y, \\
& M^{12}=\int_{\Omega_{e}}\left(I_{0} N N^{T}\right) d x d y, \\
& F=\int_{\Omega_{e}} N q d x d y-\oint_{\Gamma_{e}}\left(\frac{\partial N}{\partial n} M_{n n}+\frac{\partial N}{\partial s} M_{n s}+N Q_{n}\right)_{(18)}
\end{aligned}
$$

where $B_{1}$ and $B_{2}$ are:

$$
B_{1}=D_{1} N, \quad B_{2}=D_{2} N .
$$

\subsection{Linear rectangular element}

A non-conforming four-node rectangular element with the following six degrees of freedom per node is defined for discretizing the plate domain:

$$
\text { DOFs : }\left\{\begin{array}{llllll}
w_{b} & \frac{\partial w_{b}}{\partial x} & \frac{\partial w_{b}}{\partial y} & w_{s} & \frac{\partial w_{s}}{\partial x} & \frac{\partial w_{s}}{\partial y}
\end{array}\right\} \text {. }
$$

If $a_{e}^{b}$ and $a_{e}^{s}$ denote elemental bending and shear DOFs and $a_{i}^{b}$ and $a_{i}^{s}$ denote bending and shear nodal DOFs, we can write that:

$$
\begin{gathered}
a_{e}^{b}=\left\{\begin{array}{c}
a_{i}{ }^{b} \\
a_{j}{ }^{b} \\
a_{k}{ }^{b} \\
a_{l}{ }^{b}
\end{array}\right\} \text { where }: a_{i}^{b}=\left\{\begin{array}{c}
w_{b} \\
\partial w_{b} / \partial y \\
-\partial w_{b} / \partial x
\end{array}\right\}, \\
a_{e}^{s}=\left\{\begin{array}{c}
a_{i}{ }^{s} \\
a_{j}{ }^{s} \\
a_{k}{ }^{s} \\
a_{l}{ }^{s}
\end{array}\right\} \text { where: } a_{i}^{s}=\left\{\begin{array}{c}
w_{s} \\
\partial w_{s} / \partial y \\
-\partial w_{s} / \partial x
\end{array}\right\} .
\end{gathered}
$$

The shape functions for discretizing bending and shear components of transvers deflection are defined as follows [30]:

$$
\begin{aligned}
& N^{T}=\left[\begin{array}{lllll}
\varphi_{1}^{e} & \varphi_{2}^{e} & \varphi_{3}^{e} & \ldots & \varphi_{12}^{e}
\end{array}\right], \\
& \varphi_{i}^{e}=g_{i 1}(i=1,4,7,10),
\end{aligned}
$$

where:

$$
\begin{aligned}
& g_{i 1}=\frac{1}{8}\left(1+\xi_{0}\right)\left(1+\eta_{0}\right)\left(2+\xi_{0}+\eta_{0}-\xi^{2}-\eta^{2}\right), \\
& \varphi_{i}^{e}=g_{i 2}(i=2,5,8,11),
\end{aligned}
$$

where:

$$
\begin{aligned}
& g_{i 2}=\frac{1}{8} \xi_{1}\left(\xi_{0}-1\right)\left(1+\eta_{0}\right)\left(1+\xi_{0}\right)^{2}, \\
& \varphi_{i}^{e}=g_{i 3}(i=3,6,9,12),
\end{aligned}
$$

where:

$$
\begin{aligned}
& g_{i 3}=\frac{1}{8} \eta_{1}\left(\eta_{0}-1\right)\left(1+\xi_{0}\right)\left(1+\eta_{0}\right)^{2}, \\
& \xi=\frac{\left(x-x_{c}\right)}{a}, \quad \eta=\left(y-y_{c}\right) / b, \quad \xi_{0}=\xi \xi_{i}, \quad \eta=\eta \eta_{i} .
\end{aligned}
$$

As illustrated in Figure 2, $a$ and $b$ are half-width and half-length of element, and $x_{c}$ and $y_{c}$ are the coordinates of the mid-point of the element. 


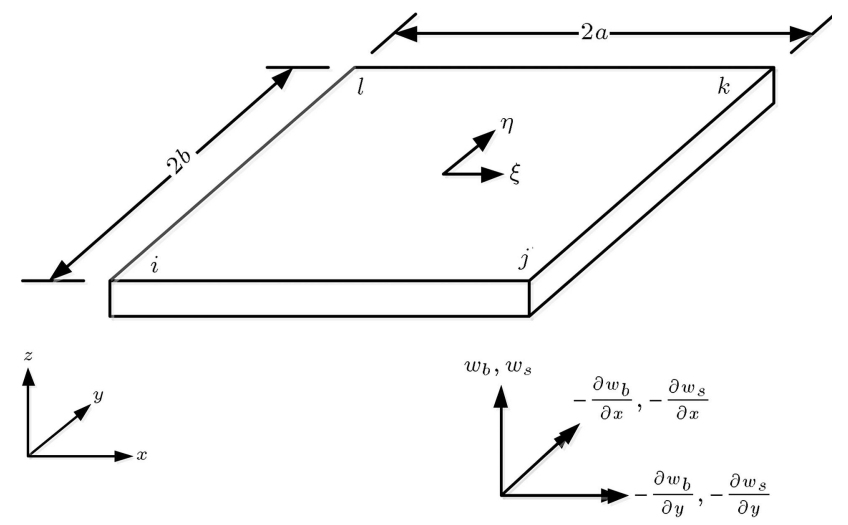

Figure 2. Rectangular plate element.

\subsection{Newmark formulation for temporal discretization}

By ignoring the damping effects, the following hyperbolic differential equation can be written for elastodynamic behavior of many engineering structures:

$$
M \frac{d^{2} u}{d t^{2}}+K u=F
$$

where $M$ and $K$ are the mass and stiffness matrices, respectively, and $F$ is the external force vector. The dynamic response of structure is solved using the wellknown Newmark-beta method. The constant average acceleration method, which is unconditionally stable for linear problems, is adopted in this research [31].

\section{Results and discussions}

A FE code based on the presented formulation is generated using MATLAB software. Some benchmark problems are solved by the code, and the obtained results are compared with the existing results in the literature.

Example 1. Consider a simply supported square plate subjected to a uniformly distributed load of $p_{0}=10 \mathrm{lbf} / \mathrm{ft}^{2}$ which is suddenly applied to plate and remains constant during time. The material and geometric parameters are considered as:

Young's modulus $E=10 \times 10^{6} \mathrm{psi}$,

Poisson's ratio $\quad \mu=0.23$,

Weight density $\rho=157.5 \mathrm{lb} / \mathrm{ft}^{3}$,

Thickness $\quad h=0.25 \mathrm{in}$, side length,

Width $\quad a=b=8 \mathrm{ft}$.

The static response of plate can be obtained analytically using Navier solution. In this method, two trigonometric series are used for independent variables $w_{b}$ and $w_{s}$ as in Eqs. (24) and (25):

$$
\begin{aligned}
& w_{b}(x, y)=\sum_{m=1}^{\infty} \sum_{n=1}^{\infty} w_{m n}^{b} \sin \left(\frac{m \pi x}{a}\right) \sin \left(\frac{n \pi y}{b}\right) \\
& w_{s}(x, y)=\sum_{m=1}^{\infty} \sum_{n=1}^{\infty} w_{m n}^{s} \sin \left(\frac{m \pi x}{a}\right) \sin \left(\frac{n \pi y}{b}\right)
\end{aligned}
$$

These expressions satisfy boundary conditions, automatically. The distributed load is estimated as double Fourier expansion series according to Eq. (26):

$$
q(x, y)=\sum_{m=1}^{\infty} \sum_{n=1}^{\infty} q_{m n} \sin \left(\frac{m \pi x}{a}\right) \sin \left(\frac{n \pi y}{b}\right) .
$$

Coefficient $q_{m n}$ is obtained by:

$$
q_{m n}=\int_{0}^{a} \int_{0}^{b} q(x, y) \sin \left(\frac{m \pi x}{a}\right) \sin \left(\frac{n \pi y}{b}\right) d x d y .
$$

By substituting displacement and load functions into governing Eqs. (9) and (10), the following relations are obtained:

$$
\begin{aligned}
& w_{m n}^{b}=\frac{q_{m n}}{D \pi^{4}\left(m^{2} / a^{2}+n^{2} / b^{2}\right)^{2}}, \\
& w_{m n}^{s}= \\
& \frac{q_{m n}}{\frac{D \pi^{4}}{84}\left(m^{2} / a^{2}+n^{2} / b^{2}\right)^{2}+\frac{5 G h \pi^{2}}{6}\left(m^{2} / a^{2}+n^{2} / b^{2}\right)},
\end{aligned}
$$

where $D$ and $G$ are defined as follows:

$$
D=\frac{E h^{3}}{12\left(1-\nu^{2}\right)}, \quad G=\frac{E}{2(1+\nu)} .
$$

Static displacement of central point of plate can be obtained analytically by substituting Eqs. (28) and (29) into Eqs. (24) and (25) and taking into account sufficient number of terms of series to fulfill converged result:

$$
\begin{aligned}
w(4,4) & =w_{b}(4,4)+w_{s}(4,4)=1.7869+.00000622 \\
& =1.7869062 \mathrm{in}
\end{aligned}
$$

The static analysis of problem is also performed by Static FF-RPT code. The effect of number of elements on central deflection of the plate is investigated in Table 1. By increasing the number of elements, the obtained results converge to analytical value. According to this table, considering 10 elements in each plate side is sufficient to achieve converged result.

The dynamic response of the plate under prescribed loading is performed using Dynamic FE-RPT code. The computational time is considered $0.15 \mathrm{~s}$ which is divided into 1500 time steps of $0.1 \mathrm{~ms}$; the obtained transverse deflection of central point of the 
Table 1. Convergence study for Example 1.

\begin{tabular}{cc}
\hline Mesh design & $\boldsymbol{w}($ in $)$ \\
\hline $2 \times 2$ & 1.248 \\
$4 \times 4$ & 1.531 \\
$6 \times 6$ & 1.684 \\
$8 \times 8$ & 1.722 \\
$10 \times 10$ & 1.734 \\
$12 \times 12$ & 1.736 \\
\hline
\end{tabular}

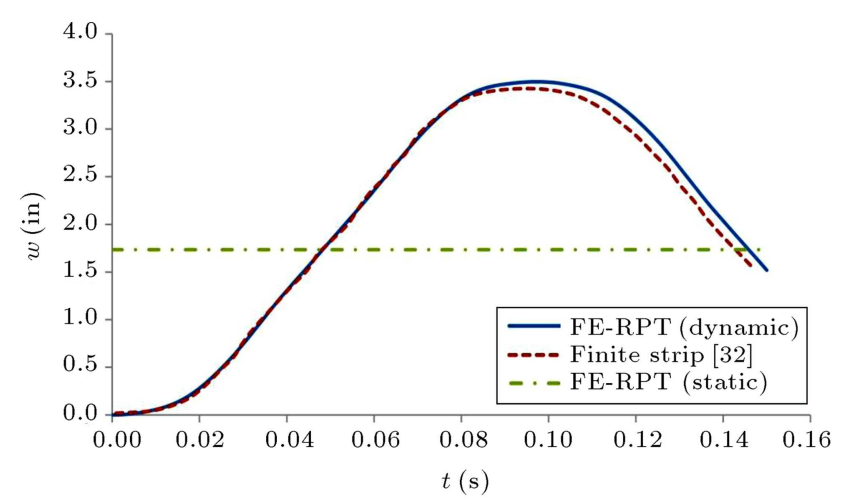

Figure 3. Comparison of central deflection of square plate subjected to uniform loading.

plate is shown in Figure 3. It is seen that the results of the presented formulation are in good agreement with those of finite strip method reported in [32], and the maximum difference between these two methods is less than 10\%. Furthermore, as expected, for the applied loading, the dynamic response of plate oscillates between 0 and twice the static value.

Example 2. Consider a simply supported rectangular plate under uniformly distributed patch load of $p_{0}=$ 0.01 psi, as shown in Figure 4. The load is suddenly applied to the patch area and remains constant during applied time. The geometry and material parameters are considered as:

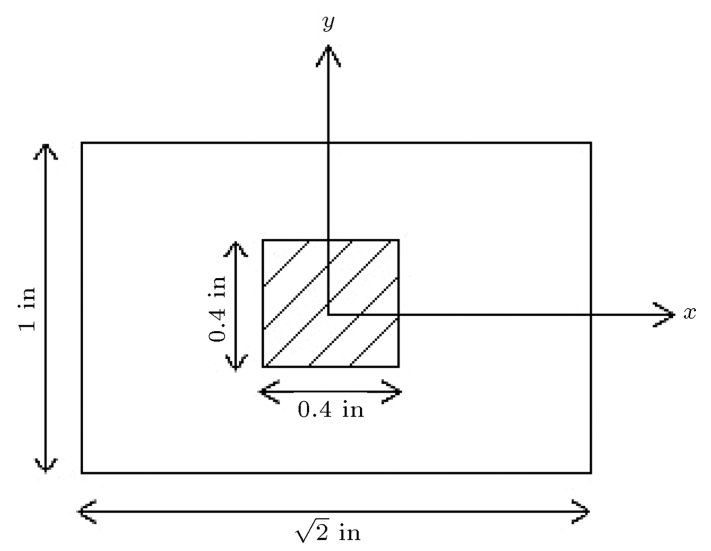

Figure 4. Rectangular plate subjected to suddenly applied patch load [32].

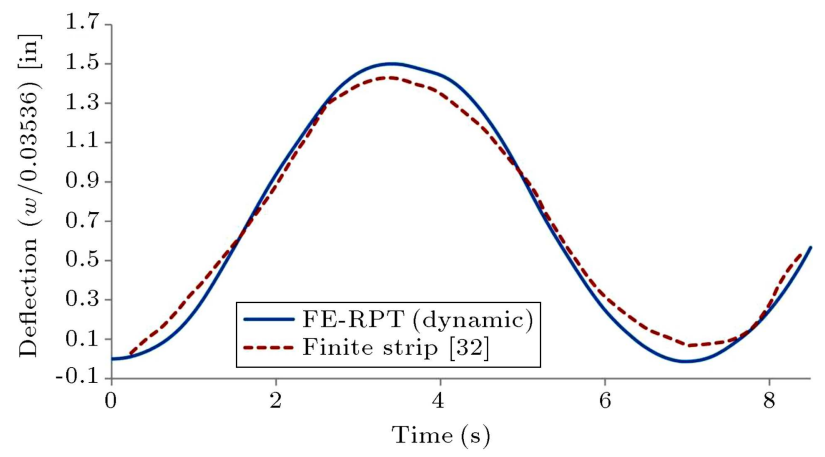

Figure 5. Time history of deflection of central point of rectangular plate subjected to suddenly applied patch load.

Young's modulus $E=1 \mathrm{lb} / \mathrm{in}^{2}$,

Poisson's ratio $\quad \mu=0.3$,

Mass density $\quad \rho=1 \mathrm{lb} \cdot \sec ^{2} / \mathrm{in}^{4}$,

Thickness $\quad h=0.2 \mathrm{in}$.

Figure 5 compares the time history of transverse deflection of central point of the plate obtained by the proposed Dynamic FE-RPT formulation with the result of finite strip method, and good agreement is observed between the results of two methods. The analysis is performed by considering time step of $1 \mathrm{~ms}$, and each plate side is discretized into 10 elements.

Example 3. A simply supported rectangular plate of $(60$ in $\times 40$ in $\times 1$ in) with material properties of $E=30 \times 10^{6}$ psi, $\mu=0.3$, and $\rho=0.00073$ lb.sec ${ }^{2} / \mathrm{in}^{4}$ is considered. The plate is subjected to a uniform pressure having triangular time variations as shown in Figure 6(a). The dynamic response of the plate is obtained using the dynamic FE-RPT code considering a time step of $0.01 \mathrm{~ms}$. Due to the symmetry of geometry and loading, half plate is discretized by a mesh of $12 \times 8$. Figure $6(\mathrm{~b})$ compares the obtained dynamic response of plate with the results of boundary element method reported in [33], showing good agreement between the results.

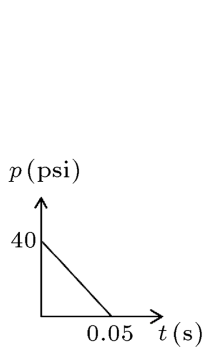

(a)

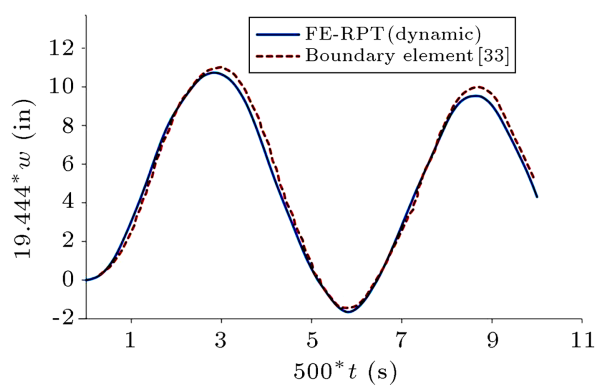

(b)
Figure 6. Central deflection of rectangular plate subjected to a uniform pressure having triangular time variation. 
Table 2. Material properties of orthotropic plate.

\begin{tabular}{cccccccc}
\hline $\boldsymbol{E}_{\mathbf{1}}(\mathbf{G P a})$ & $\boldsymbol{E}_{\mathbf{2}} / \boldsymbol{E}_{\mathbf{1}}$ & $\boldsymbol{G}_{\mathbf{1 2}} / \boldsymbol{E}_{\mathbf{1}}$ & $\boldsymbol{G}_{\mathbf{1 3}} / \boldsymbol{E}_{\mathbf{1}}$ & $\boldsymbol{G}_{\mathbf{2 3}} / \boldsymbol{E}_{\mathbf{1}}$ & $\boldsymbol{\mu}_{\mathbf{1 2}}$ & $\boldsymbol{\mu}_{\mathbf{2 1}}$ & $\boldsymbol{\rho}\left(\mathrm{kg} / \mathbf{m}^{\mathbf{3}}\right)$ \\
\hline 200 & 0.525 & 0.26293 & 0.15991 & 0.26293 & 0.44026 & 0.23124 & 7860 \\
\hline
\end{tabular}

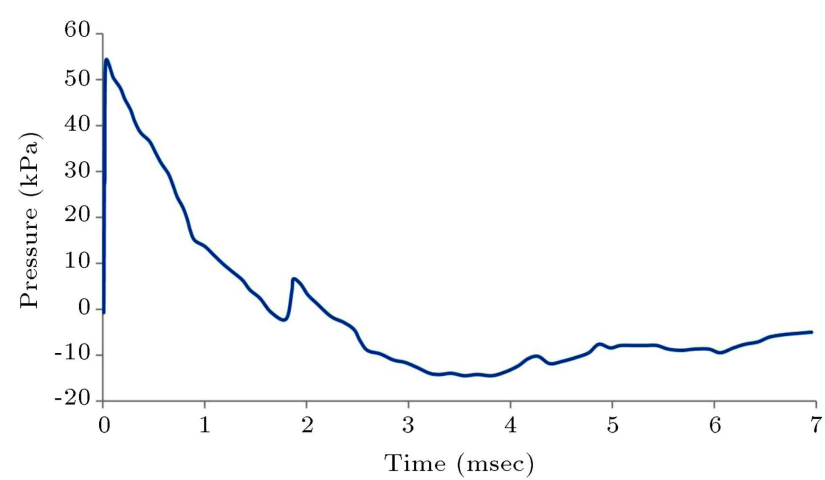

Figure 7. Time variation of air blast pressure acting on a square plate [34].

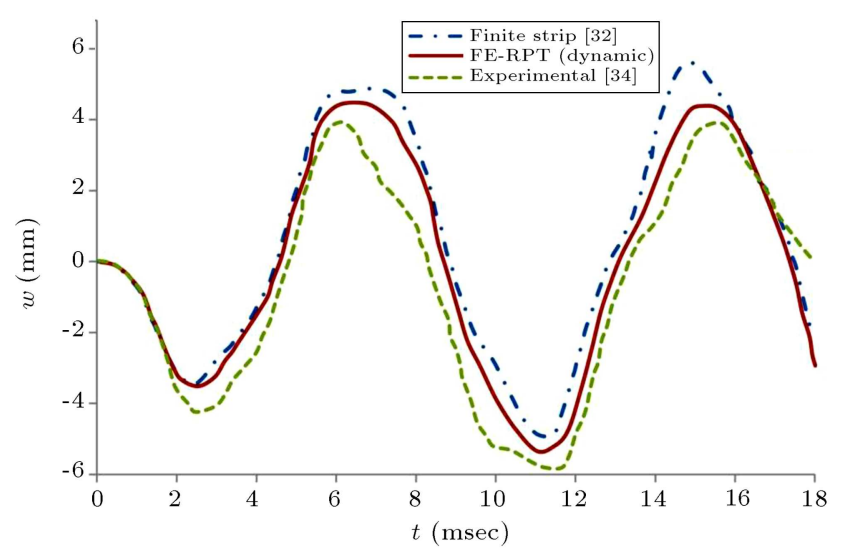

Figure 8. Time history of central deflection of square plate subjected to air blast.

Example 4. A square plate with length of $508 \mathrm{~mm}$, thickness of $3.4 \mathrm{~mm}$, and material properties of $E=$ $206.84 \mathrm{GPa}, \mu=0.3$, and $\rho=7900 \mathrm{~kg} / \mathrm{m}^{3}$ is considered. All edges are clamped, and the plate is subjected to air blast pressure shown in Figure 7 . The obtained time history of central deflection of plate is compared with experimental [34] and finite strip method [32] results in Figure 8, where good agreement is observed between the results. A mesh of $12 \times 12$ is used for discretization of half plate, and time step is set to $0.01 \mathrm{~ms}$.

\subsection{Parametric study}

In this section, an orthotropic plate under uniformly distributed loading with various boundary conditions, including fully simply support (SSSS), fully clamp (CCCC), and simply-clamp (SSCC), is considered, and effects of different parameters, such as aspect ratios, orthotropy ratio $\left(E_{1} / E_{2}\right)$, and thickness ratio, are studied on normalized transverse deflections, in-plane normal stresses, and transverse shear stresses. The

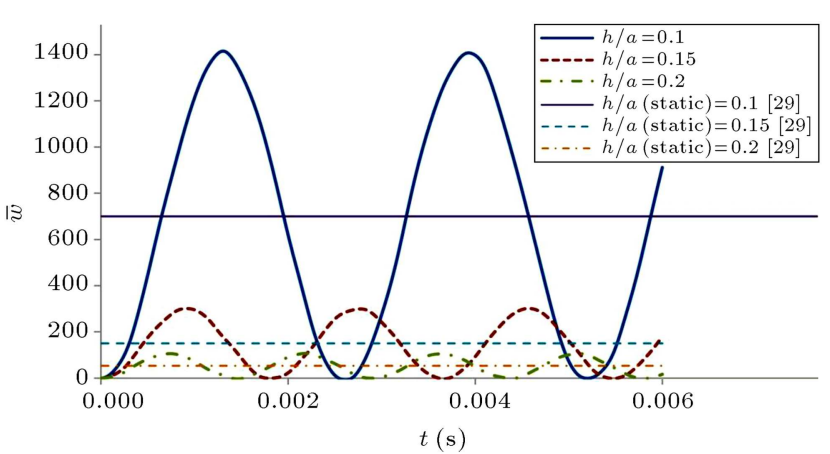

Figure 9. Normalized central deflection of orthotropic plate considering different thickness ratios, where $a / b=1$.

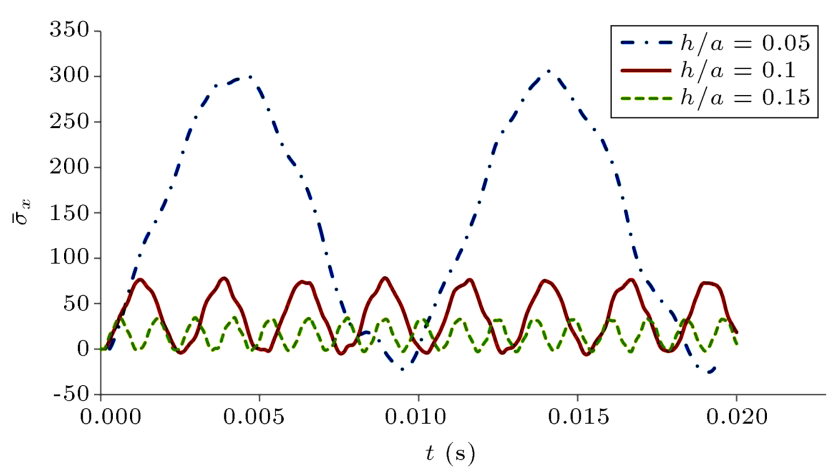

Figure 10. Normalized central stress, $\bar{\sigma}_{x}$, of orthotropic plate considering different thickness ratios, where $a / b=1$.

analyses are performed using a mesh of $10 \times 10$ for the half plate and a time step of $0.01 \mathrm{~ms}$. Material properties are chosen according to Table 2, and the following normalized parameters are introduced as follows:

$$
\begin{aligned}
& \bar{w}=w Q_{11} / h q_{0}, \quad \bar{\sigma}_{x}=\sigma_{x} / q_{0}, \\
& \bar{\sigma}_{y}=\sigma_{y} / q_{0}, \quad \bar{\tau}_{x z}=\tau_{x z} / q_{0} .
\end{aligned}
$$

Figures 9 and 10 show the normalized transverse deflection and normal stress of plate for different thickness ratios. As seen, the dynamic responses oscillate between zero and twice the static values, and the increment of the thickness ratio increases the stiffness of plate, which results in the increase of frequency and decrease of amplitude of results.

The effect of boundary condition on normalized transverse deflection and shear stress is investigated, as shown in Figures 11 and 12. As expected, by changing boundary conditions from fully simply supported to fully clamped and, consequently, increasing the constraints on plate, the amplitude and frequency of results decrease and increase, respectively. 


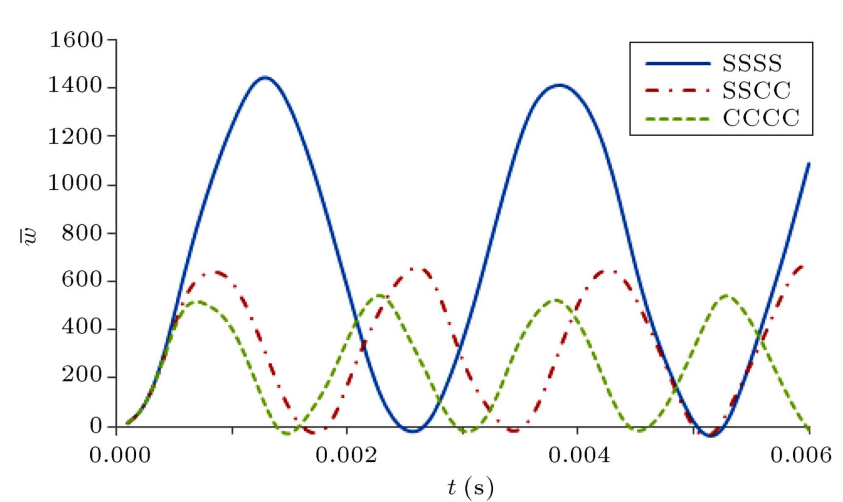

Figure 11. Normalized central deflection of orthotropic plate considering different boundary conditions, where $h / a=0.1$ and $a / b=1$.

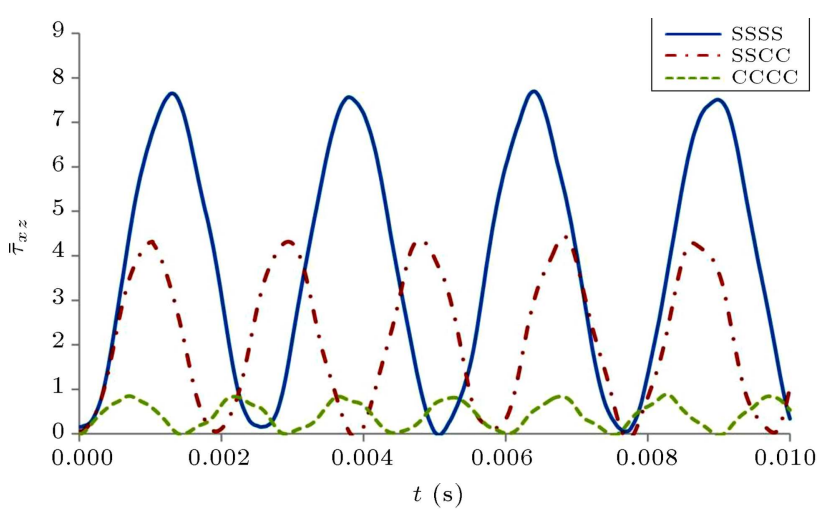

Figure 12. Normalized central out-of-plane shear stress, $\bar{\tau}_{x z}$, of orthotropic plate considering different boundary conditions, where $h / a=0.1$ and $a / b=1$.

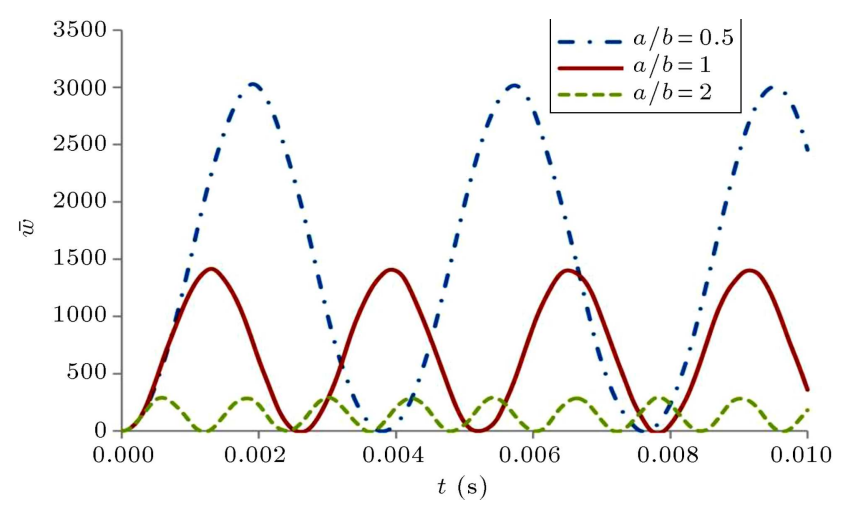

Figure 13. Normalized central deflection of orthotropic plate considering different aspect ratios, where $h / a=0.1$.

In Figures 13-15, effects of plate aspect ratio on normalized deflections and stresses are studied. Parameter $a$ is constant in all analyses; by increasing $a / b$ ratio and, consequently, decreasing the plate dimension $(b)$, frequency and amplitude of results obviously increase and decrease, respectively.

The effect of orthotropy ratio $\left(E_{1} / E_{2}\right)$ is investigated on dynamic response of plate in Figures 16 and 17. Parameter $E_{1}$ is kept constant in all anal-

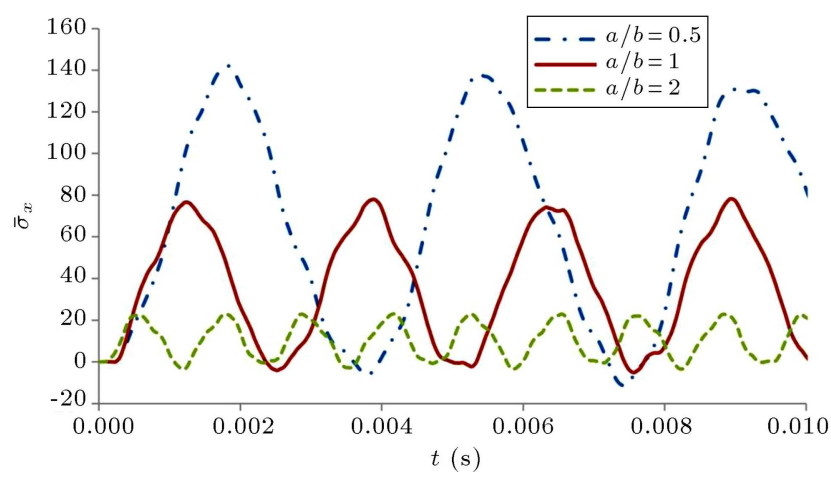

Figure 14. Normalized central normal stress, $\overline{\sigma_{x}}$, of orthotropic plate considering different aspect ratios, where $h / a=0.1$.

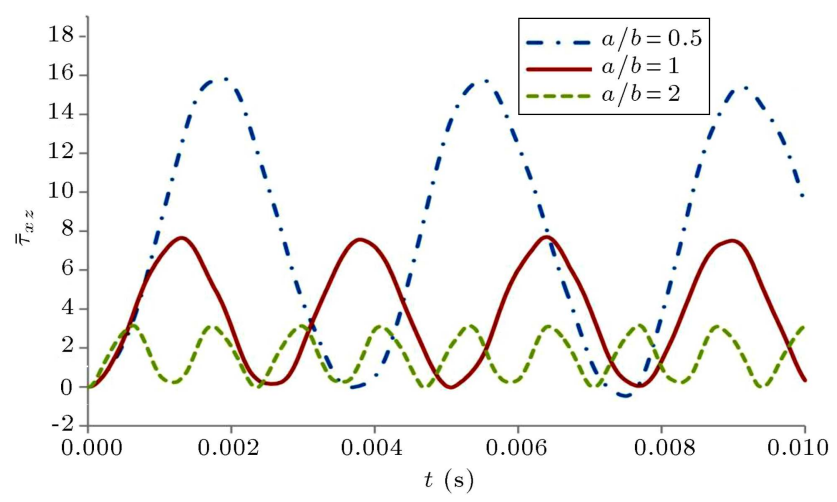

Figure 15. Normalized central out-of-plane shear stress, $\bar{\tau}_{x z}$, of orthotropic plate considering different aspect ratios, where $h / a=0.1$.

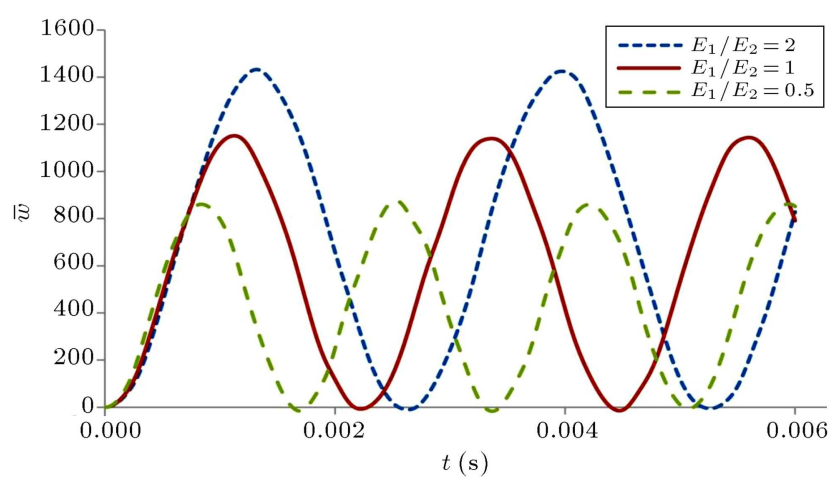

Figure 16. Normalized central deflection of orthotropic plate considering different orthotropy ratios, where $h / a=0.1$ and $a / b=1$.

yses. Therefore, by increasing $E_{1} / E_{2}$ ratio, $E_{2}$ and, consequently, the overall stiffness of structure decrease; moreover, frequency and amplitude of $\bar{\sigma}_{x}$ and $\bar{\tau}_{x z}$ decrease and increase, respectively.

\section{Conclusion}

In this study a finite-element formulation for dynamic analysis of isotropic and orthotropic plates is developed 


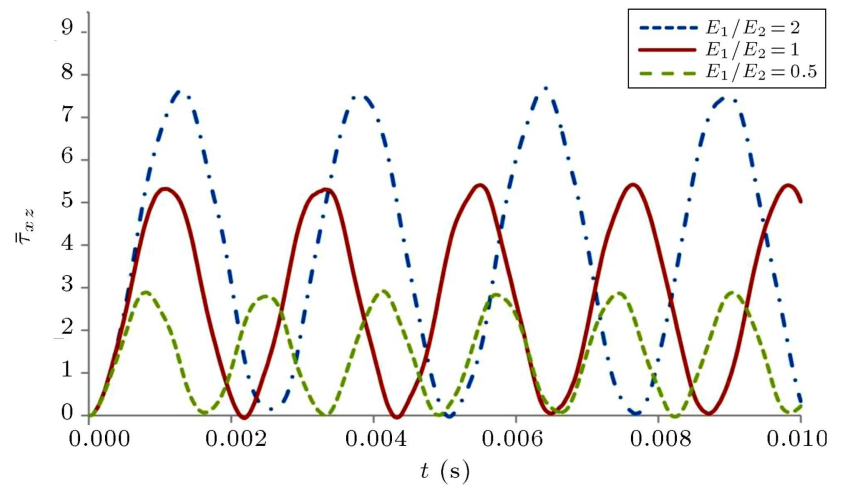

Figure 17. Normalized central out-of-plane shear stress, $\bar{\tau}_{x z}$, of orthotropic plate considering different orthotropy ratios, where $h / a=0.1$ and $a / b=1$.

using the two-variable refined plate theory. This theory, which can be used for both thin and thick plates, predicts parabolic variation of transverse shear stresses across the plate thickness. In comparison with FSDT with three unknown parameters and the HSDTs with more than three parameters, this theory has only two parameters; therefore, the simplicity and accuracy are the main features of the present formulation. The main novelty of this work is constructing a dynamic finite-element model based on the two-variable refined plate theory for analysis of plate problems.

After constructing weak-form equations using the Hamilton's principle, a new 4-node rectangular plate element with six degrees of freedom at each node is introduced for discretization of the domains. The semidiscrete approach is adopted to deal with the dynamic analysis, and an unconditionally stable implicit Newmark scheme is used for temporal discretization. The efficiency and accuracy of the presented formulation are proved by solving some benchmark isotropic and orthotropic plate problems. The obtained results are in good agreement with analytical solutions of common plate theories. Moreover, the effects of aspect ratio, thickness-to-side ratio, material properties and type of boundary conditions on deflections and stresses are investigated.

The results show that the two-variable refined plate theory is simple and efficient in comparison with previous higher-order shear deformation plate theories; moreover, the present finite-element formulation can be a very useful tool for simulation of both thin and thick plate problems under static and dynamic loadings. The present study can be extended to other plate structures, such as functionally graded, laminated composite, and smart plates.

\section{References}

1. Kirchhoff, G. "Equilibrium and motion of an elastic disc [ÄUber das gleichgewicht und die bewegung einer elastischen scheibe", J. ReineAngew. Math., 1859(40), pp. 51-88 (1850).

2. Reissner, E. "The effect of transverse shear deformation on the bending of elastic plates", J. Appl. Mech., 12(2), pp. 69-77 (1945).

3. Mindlin, R.D. "Influence of rotary inertia and shear on flexural motions of isotropic elastic plates", J. Appl. Mech., 18(1), pp. 31-38 (1951).

4. Whitney, J.M. and Sun, C.T. "A higher order theory for extensional motion of laminated composites", Sound Vib., 30(1), pp. 85-97 (1973).

5. Hanna, N.F. and Leissa, A.W. "A higher order shear deformation theory for the vibration of thick plates", Sound Vib., 170(4), pp. 545-555 (1994).

6. Reddy, J.N. "A simple higher-order theory for laminated composite plates", ASME. J. Appl. Mech., 51(4), pp. 745-752 (1984).

7. Reddy, J.N. and Phan, N.D. "Stability and vibration of isotropic, orthotropic and laminated plates according to a higher-order shear deformation theory", Sound $V i b ., 98(2)$, pp. 157-170 (1985).

8. Bhimaraddi, A. and Stevens, L.K. "A higher order theory for free vibration of orthotropic, homogeneous, and laminated rectangular plates", J. Appl. Mech., 51(1), pp. 195-198 (1984).

9. Kant, T. "Numerical analysis of thick plates", Comput. Methods Appl. Mech. Eng., 31(1), pp. 1-18 (1982).

10. Lo, K.H., Christensen, R.M., and Wu, E.M. "A highorder theory of plate deformation, Part 1: Homogeneous plates", J. Appl. Mech., 44(4), pp. 663-668 (1977).

11. Ghugal, Y.M. and Sayyad, A.S. "A static flexure of thick isotropic plates using trigonometric shear deformation theory", J. Solid. Mech., 2(1), pp. 79-90 (2010).

12. El Meiche, N., Tounsi, A., Ziane, N., Mechab, I., and Adda Bedia, E.A. "A new hyperbolic shear deformation theory for buckling and vibration of functionally graded sandwich plate", Int. J. Mech. Sci., 53(4), pp. 237-247 (2011).

13. Shimpi, R.P. "Refined plate theory and its variants", AIAA J., 40(1), pp. 137-146 (2002).

14. Shimpi, R.P. and Patel H.G., "A two variable refined plate theory for orthotropic plate analysis", Int. J. Solids Struct., 43(22-23), pp. 6783-6799 (2006).

15. Kim, S.E., Thai, H.T., and Lee, J. "A two variable refined plate theory for laminated composite plates", Compos. Struct., 89(2), pp. 197-205 (2009).

16. Thai, H.T. and Kim, S.E. "Analytical solution of a two variable refined plate theory for bending analysis of orthotropic Levy-type plates", Int. J. Mech. Sci., 54(1), pp. 269-276 (2012).

17. Rouzegar, J. and Abdoli Sharifroor, R. "Finite element formulations for free vibration analysis of isotropic and orthotropic plates using two-variable refined plate theory", Sci. Iran., 23(4), pp. 1787-1799 (2016). 
18. Rouzegar, J. and Abdoli Sharifroor, R. "Finite element formulations for buckling analysis of isotropic and orthotropic plates using two-variable refined plate theory", Iran. J. Sci. Technol. - Trans. Mech. Eng., 41(3), pp. 177-187 (2017).

19. Rouzegar, J. and Abad, F. "Analysis of cross-ply laminates with piezoelectric fiber-reinforced composite actuators using four-variable refined plate theory", $J$. Theor. Appl. Mech., 53(2), pp. 439-452 (2015).

20. Rouzegar, J. and Abad, F. "Free vibration analysis of FG plate with piezoelectric layers using four-variable refined plate theory", Thin Wall. Struct., 89(1), pp. 76-83 (2015).

21. Rouzegar, J. and Gholami, M. "Non linear bending analysis of thick rectangular plates by four-variable Refined plate theory and Dynamic Relaxation method", Modares Mech. Eng., 15(2), pp. 221-230 (2015) (In Persian).

22. Nguyen-Xuan, H., Tran, L.V., Thai, C.H., Kulasegaram, S., and Bordas, S.P.A. "Isogeometric analysis of functionally graded plates using a refined plate theory", Compos. Part B-Eng., 64, pp. 222-234 (2014).

23. Thai, C.H., Kulasegaram, S., Tran, L.V., and NguyenXuan, H. "Generalized shear deformation theory for functionally graded isotropic and sandwich plates based on isogeometric approach", Comput. Struct., 141, pp. 94-112 (2014).

24. Nguyen, K.D. and Nguyen-Xuan, H. "An isogeometric finite element approach for three-dimensional static and dynamic analysis of FGM plates structures", Compos. Struct., 132, pp. 423-439 (2015).

25. Nguyen, T.N., Thai, C.H., and Nguyen-Xuan, H. "On the general framework of high order shear deformation theories for laminated composite plate structures: A novel unified approach", Int. J. Mech. Sci., 110, pp. 242-255 (2016).

26. Nguyen, L.B., Thai, C.H., and Nguyen-Xuan, H. "A generalized unconstrained theory and isogeometric finite element analysis based on Bezier extraction for laminated composite plates", Eng. Comput., 32(3), pp. 457-475 (2016).

27. Patel, H. and Shimpi, R.P. "A New Finite Element for a Shear Deformable Plate", 47th AIAA/ASME/ASCE/AHS/ASC Structures, Structural Dynamics, and Materials Conference., Newport, Rhode Island, USA (2006).
28. Katori, H. and Okada, T. "Plate element based on a higher-order shear deformation theory", Transactions of the Japan Society of Mechanical Engineers Series A, 72(716), pp. 412-418 (2006).

29. Rouzegar, J. and Abdoli Sharifroor, R. "A finite element formulation for bending analysis of isotropic and orthotropic plates based on two-variable refined plate theory", Sci. Iran., 22(1), pp. 196-207 (2015).

30. Melosh, R.J. "Structural analysis of solids", J. Struct. Div. ASCE, 89(4), pp. 205-223 (1963).

31. Rouzegar, S.J. and Mirzaei, M. "Modeling dynamic fracture in Kirchhoff plates and shells using the extended finite element method", Sci. Iran., 20(1), pp. 120-130 (2013).

32. Sheikh, A.H. and Mukhopadhyay, M. "Linear and nonlinear transient vibration analysis of stiffened plate structures", Finite Elem. Anal. Des., 38(14), pp. 477$502(2002)$.

33. Providakis, C.P. and Beskos, D.E. "Free and forced vibration of plates by boundary elements", Comput. Methods Appl. Mech. Eng., 74(3), pp. 231-250 (1989).

34. Houlston, R. and Slater, J.E. "A summary of experimental results on square plate and stiffened plates subjected to air blast loading", Shock Vibr. Bull., 2(57), pp. 14-16 (1986).

\section{Biographies}

Jafar Rouzegar is currently an Assistant Professor at the Department of Mechanical and Aerospace Engineering of Shiraz University of Technology, Iran. He received his BSc degree in Mechanical Engineering from Shiraz University, Iran in 2002. He also received his MSc and PhD degrees in Mechanical Engineering from Tarbiat Modares University, Iran in 2004 and 2010, respectively. His research interests include FEM and XFEM, theories of plates and shells, and fracture mechanics.

Mohammad Sayedain received his BSc in Mechanical Engineering from Vali-e-Asr University, Rafsanjan, Iran in 2013. He also received his MSc degree in Mechanical Engineering from Shiraz University of Technology, Iran in 2015. His research interests include FEM, theories of plates and shells and composite materials. 